Kairos. Journal of Philosophy \& Science 16, 2016 Center for the Philosophy of Sciences of Lisbon University

\title{
Leibniz crítico de Euclides. El método del Analysis Situs
}

\author{
Javier Echeverría \\ Universidad del País Vasco (UPV/EHU) \\ País Vasco (España) \\ javier.echeverria.ezponda@gmail.com \\ Mary Sol de Mora \\ Universidad del País Vasco (UPV/EHU) \\ País Vasco (España) \\ demora@leibnizsociedad.org
}

DOI 10.1515/kjps-2016-0011

\section{Introducción}

Durante su primera juventud, Leibniz leyó los Elementos de Euclides, pero no profundizó en ellos hasta años más tarde. Sabemos que la Geometría de Euclides le pareció muy complicada de entender en su primera lectura. No obstante, mucho antes de su estancia en París (1672-1676), ya en 1670, podemos encontrar algunos de los conceptos que aparecerán posteriormente en sus diversos ensayos sobre Analysis Situs. En esos años sus conocimientos de la geometría de Euclides y de matemáticas eran bastante escasos. Sorprendentemente, en ese extraordinario compendio de las ideas juveniles de Leibniz que es el suplemento de su 
tesis, el De Arte Combinatoria $\left(1666^{1}\right)$, ya encontramos alusiones interesantes al situs. Así, en el comienzo de ese texto, tras la demostración de la existencia de Dios, incluye algunas consideraciones metafísicas sobre el todo y las partes:

"8. Además, el mismo Todo (y así el Número o la Totalidad) puede romperse en partes como totales menores, éste es el fundamento de las Complexiones, de manera que se podrían entender dadas partes comunes en los mismos diversos todos menores, por ejemplo, sea el Todo A. B. C. Todos menores serían sus partes $A B, B C, A C$ : y también la disposición de sus partes mínimas, o sea de las mínimas de las supuestas (es decir, las Unidades), puede variar entre sí y con el todo, al que se Ilama situs"2.

Más adelante, en el apartado Definiciones, define el lugar de este modo:

“3. El lugar es la localización de las partes.

4. El lugar es, o bien absoluto, o bien relativo: aquel, el de las partes con el todo, éste, el de las partes con las partes. En aquel se considera el número de los lugares y la distancia del inicio al final, en éste no se atiende ni al inicio ni al final, sino que se considera la distancia de las partes a una parte dada. Por ello aquel se expresa por una línea o líneas que no cierran una figura ni vuelven sobre sí mismas y de manera óptima por una línea recta; éste por una línea o líneas que cierran una figura y de manera óptima por un círculo. En aquel, la razón de prioridad o posterioridad es considerada la más poderosa, en éste, sin importancia. Así pues, aquel debería llamarse Orden por excelencia;

5. A éste «se debería llamar Vecindad³, a aquel disposición,

a éste composición. Así pues los siguientes lugares se dif-

1 Véase M.S. de Mora. (2015), pp. 547-643.

2 Lugar o situación.

3 O proximidad. N. del Ed. 
erencian por razón de su orden: abcd, bcda, cdab, dabc. En cuanto a la Proximidad no existe ninguna variación sino que se percibe

un solo lugar, es decir, este: a c". $d$

También encontramos ya uno de sus conceptos más utilizados posteriormente en su Geometría Situs: el de homogeneidad:

"17. Cosa homogénea es la que se puede colocar igualmente en cualquier lugar dado, excepto en la parte fija”.

Y más sorprendente aún, encontramos estos comentarios sobre Euclides y sobre las nociones más simples:

"87. Muy convenientemente, la Matemática se ve actualmente como un esfuerzo extemporáneo: de aquí que no hayamos comenzado simplemente por los términos primitivos, sino por los primitivos en Matemática; pero tampoco podemos <comenzar> por todos, sino por aquellos que por su complejidad, juzguemos suficientes para producir los términos generados propuestos. Con este método hubiéramos podido exponer todas las definiciones de los Elementos de Euclides, si hubiera quedado tiempo. Ahora bien, puesto que no hemos partido simplemente de los términos primitivos, era necesario adoptar unos signos con los que se comprendieran los casos de las palabras y otras cosas necesarias para completar el discurso. Puesto que, si hubiéramos comenzado por los términos primitivos simples, en vez de por las variaciones de dichos casos, hubiésemos expuesto los términos cuyo origen expuso, Jul.Caesar Scaligero ${ }^{4}$, en el Lib. De Caus. L.L., a partir de

4 Giulio Cesare Scaligero o della Scala, latinizado Julius Caesar Scaliger y también Ilamado Julio César Escalígero (1484 - 1558), médico, filósofo, botánico y humanista italiano, la parte más fructífera de su vida transcurrió en Francia. Autor de De causis linguae latinae libri XIII. 1540. 
las relaciones y de la Metafísica. Pero nosotros en cambio, adoptaremos los artículos griegos.

88. Por tanto, sea la Clase I, en la que los términos primitivos $^{5}<$ son>: 1. Punto, 2. Espacio, 3. Colocado entre, 4. Adjunto o Contiguo, 5. Diseminado o Distante, 6. Final, o sea que dista, 7. Ínsito, 8. Incluido (por ejemplo, el centro está ínsito en el círculo, incluido en la circunferencia), 9. Parte, 10. Todo, 11. Idéntico, 12. Diverso, 13. Uno, 14. Número, 15. Varios, por ejemplo, 1.2.3.4.5, etc. 16. Distancia. 17. Posible, 18. Universo, 19. Dado, 20. Hecho, 21. Dirección, 22. Dimensión, 23. Largo, 24. Ancho, 25. Profundo, 26. Común, 27. En Progresión, o sea, continuado".

Resumimos aquí el resto de las clases que propone, eliminando la notación:

"Clase II. 1. Ia cantidad. 2. Incluyente. Clase III. 1 Intervalo . 2. Igual a. 3. Continuo. Clase IV. 1. A es Mayor. 2. Menor, 3. Línea, 4 Paralela 5. Figura. Clase V. 1. Creciente 2. Decreciente, 3. Implicado. 4 Secante. Clase VI. 1. Convergente, 2. Divergente. Clase VII. 1. Superficie, . 2. Infinito, 3. Periferia. 4. Medida de. Clase VIII. 1. Máximo. 2. Mínimo, 3. Recta,. 4. la que no es tal, es Curva. 5. Arco. Clase IX. 1. Área. Clase X. 1. Conmensurables. Clase XI. 1. Ángulo. Clase XII. 1. Plano. Clase XIII. 1. Gibosidad. Clase XIV. 1. <superficie> Rectilínea . 2. Ios cuales se Ilaman Lados. 3. Triángulo. 4. Cuadrángulo, etc. Clase XV. 1. LúnuIa. Clase XVI. 1. Ángulo recto. 2. Segmento. Clase XVII. 1. $<$ triángulo> Equilátero 2. triángulo Isósceles 3. <triángulo> Escaleno. Clase XVIII. 1. Ángulo de contacto. Clase XIX. 1. $<$ figura> Inscrita 2. Circunscrita. Clase XX. Ángulo Obtuso. Clase XXI. 1. Diámetro. Clase XXII. 1. Círculo 2. triángulo Rectángulo. Clase XXIII 1. Centro de una figura. Clase XXIV.

$5 \quad$ Estos términos primitivos todavía revelan que el limitado conocimiento de Leibniz de las matemáticas se reduce a los Elementos de Euclides. 
1. Semifigura dada, (por ejemplo, un semicírculo, etc.)".

Matemática y Metafísica ya están para Leibniz en relación íntima:

“9. Así aparecen dos géneros de Variaciones $^{6}$, las Complexiones y el Lugar. Y tanto la Complexión como el Lugar pertenecen a la Metafísica, es decir, a la doctrina del Todo y de las partes, si son considerados en sí mismos.

Si realmente observásemos la Variabilidad, es decir la cantidad de variación, se estaría llegando a los números y a la Aritmética. Pues tendería a creer que la doctrina de la Complexión pertenece más a la Aritmética pura, el lugar a la figurada, pues así las unidades son entendidas como formando la línea. Aunque quiero hacer notar aquí que las unidades se pueden disponer al modo de la línea recta, o del círculo, o de otras líneas, o de líneas que retornan sobre sí mismas, o figuras que cojean, en el primer modo en un lugar absoluto, o sea de partes respecto al todo, Orden; en los modos posteriores, en un lugar relativo o de partes de partes, Vecindad ${ }^{7}$ que diremos cómo difieren más abajo, en las definiciones 4. y 5 . Aquí basta con este proemio, de forma que queda de manifiesto en qué disciplina tienen su sede estas materias.

$6 \quad$ Aquí utiliza Leibniz la palabra variaciones en el sentido vulgar, no como un concepto matemático, en el que las variaciones de $m$ elementos tomados de $n$ en $n$ serían las que tienen en cuenta el lugar, a diferencia de las complexiones (combinaciones) que sólo tienen en cuenta las cosas en cualquier orden.

7 El texto de Leibniz sobre el Situs en De Arte Combinatoria es realmente notable, sobre todo en esta segunda acepción. Aquí introduce la noción de vecindad (los entornos topológicos son denominados en francés voisinages) y la idea de Leibniz es la de un entorno (circular, como pone por ejemplo, pero valdría para cualquier otra figura convexa y cerrada), de un todo que está formado por las partes de dicho todo, que están incluidas en, y por ende son partes de dicha figura convexa y cerrada. No es la noción de entorno topológica actual, pero sí una noción conjuntista actual. Por otra parte, vemos que Leibniz ya tiene la idea de juntar un punto con otro punto por medio de diversos tipos de líneas (los rígidos conectantes de 1679), de las cuales la más simple es la recta. Podríamos decir que la idea de la conexión por arcos (en el sentido actual del término) está ya intuida en 1666, y luego la desarrolló en 1679. Este punto de la conexión por arcos era uno de los argumentos de J. Echeverría en su tesis de Estado a favor de Leibniz como precedente de la topología. Lo extraordinario es que ahora ya se ve en 1666. 
3. El lugar es la localización de las partes.

4. El lugar es o bien absoluto o bien relativo: aquel, el de las partes con el todo, éste, el de las partes con las partes. En aquel se considera el número de los lugares y la distancia del inicio al final, en éste no se atiende ni al inicio ni al final, sino que se considera la distancia de las partes a una parte dada. Por ello aquel se expresa por una línea o líneas que no cierran una figura ni vuelven sobre sí mismas y de manera óptima por una línea recta; éste por una línea o líneas que cierran una figura y de manera óptima por un círculo. En aquel, la razón de prioridad o posterioridad es considerada la más poderosa, en éste, sin importancia. Así pues, aquel debería Ilamarse Orden por excelencia."

\section{Leibniz, lector y comentarista de Euclides.}

A finales del siglo XVI y durante el siglo XVII, se publicaron numerosas ediciones de los Elementos de Euclides, tanto en latín como en otras lenguas europeas. Algunos de esos editores revisaron las demostraciones de Euclides, por ejemplo Clavius, el director del Colegio Romano, quien presentó los razonamientos de Euclides en forma de silogismos (Euclides 1591). Leibniz comenzó a estudiar a fondo los Elementos de Euclides, en 1663 , a los 17 años, por consejo de J. Kühn ${ }^{8}$, aunque es posible que la idea de reformular el texto de Euclides con mayor rigor lógico le fuese sugerida por su profesor en Jena, Erhard Weigel, quien acababa de publicar un libro sobre las demostraciones aristotélico-euclídeas (Weigel 1662). Es asimismo verosímil que a través de Weigel conociese las críticas de Pierre de la Ramée a Euclides ${ }^{9}$, puesto que Weigel se declaraba contrario a las ideas de Ramus ${ }^{10}$. También la influencia de Hobbes parece

8 Véase Hofmann (1949) y (1966, p. 422).

9 Véase Arithmeticae libri duo, Basileae 1569 y Scholarum Mathematicarum libri 31, Francofurti 1637.

10 Véase Weigel, Idea Totius Encyclopediae Mathematico-Philosophici, Jenae, 1671, pp. 161-167. Petrus Ramus fue Rector de la Sorbona y Leibniz conoció bien sus obras durante su posterior estancia en París (1672-1676). 
haber sido importante en relación con las demostraciones de Euclides ${ }^{11}$. Durante toda su vida, Leibniz mantuvo firme esta convicción y retomó sus lecturas y ensayos sobre Euclides continuadamente, aunque no publicó sus resultados. Por eso hay que rastrear sus aportaciones a Euclides a través de innumerables manuscritos y algunas cartas.

Tras su llegada a París, le propuso a Gallois una demostración del axioma del todo y de las partes sobre la cual volvió en varias ocasiones ${ }^{12}$. En invierno de 1674-75 encontró por fin el tiempo necesario para leer más atentamente los Elementos ${ }^{13}$. En esos años, al parecer, se produjo una extraordinaria conjunción entre ambos proyectos: la posible demostración de los axiomas de Euclides y la construcción de una nueva Característica Geométrica, es decir, un nuevo análisis formal de la Geometría basado en la situación. En 1677 Leibniz escribió un primer ensayo sobre la Characteristica Geometrica, donde expuso su proyecto de construir un nuevo Análisis Geométrico diferente de los de Euclides, Arquímedes, Viète y Descartes ${ }^{14}$. En aquel momento, el Analysis Situs de Leibniz y la lectura crítica de Euclides formaron parte del mismo proyecto: es necesario partir de los Elementos para intentar superarlos, ampliar la Geometría de las magnitudes y construir una Geometría de las Situaciones. Para ello, hay que proponer nuevas definiciones, nociones, axiomas, problemas, teoremas y demostraciones geométricas, introduciendo nuevos signos o caracteres para expresar el corpus euclídeo y conseguir reducirlo a un Cálculo Geométrico ${ }^{15}$.

11 En el ejemplar del De Corpore conservado en Hannover (1655, p. 60) Leibniz escribió en el margen 'Definitiones' y a continuación subrayó estos pasajes de Hobbes: "Praeter definitiones, alia propositio nulla dicenda Prima est, neque ergo si paulo severius agere volumus in numerum Principiorum ascribenda est: nam Axiomata quae habentur apud Euclidem, quia demonstrari possunt, Principia demonstrandi non sunt" (...) "Deinde ea quae Postulata \& Petitiones appellantur, Principia quidem revera sunt, non tamen demonstrationis, sed constructionis" (Hobbes, Elementorum Philosophiae sectio Prima De Corpore, Londini, 1655 (P-A 743).

12 Véase, por ejemplo, la edición Gerhardt de los Philosophische Schriften, III, 321-2; V, 207 y 395-6; VII, 20 y 273-4.

13 Leibniz utilizó sobre todo las ediciones de Clavius y de Barrow, cuyos ejemplares conservados en las bibliotecas de Hannover y Wolfenbüttel conservan sus notas marginales.

14 Ver Leibniz 1995, fragmento II, pp. 50-65.

15 Al respecto, ver la introducción de J. Echeverría a Leibniz (1995), así como Echeverría (1984). 
A pesar de la adversa recepción por parte de Huygens ${ }^{16}$ con respecto a este proyecto, Leibniz continuó trabajando en él. En una comunicación presentada al $V$. Internationaler Leibniz-Kongress ${ }^{17}$, J. Echeverría propuso una primera clasificación de las nueve épocas en las que Leibniz se ocupó del l'Analysis Situs y en ocasiones de la demostración de los axiomas de Euclides. En el presente artículo se subrayarán los fragmentos más notables desde el punto de vista de la revisión de Euclides y se encuadrará este género de investigación leibniziana en el panorama científico de su época.

\section{Revisión de los Elementos}

Es bien sabido que durante su estancia en París (1672-1676), Leibniz se interesó por los intentos de Roberval de demostrar los axiomas de Euclides (Hoffmann 1974, Taton 1978). Por nuestra parte, hemos comprobado que Leibniz leyó con atención la mayor parte de las ediciones de Euclides, como lo corroboran las notas marginales que puso en algunos ejemplares de los Elementos conservados en Hannover y en Wolfenbüttel, así como sus citas al libro de Euclides en numerosos manuscritos suyos, buena parte de los cuales permanecen inéditos. En ellos proponía correcciones al texto de los Elementos y demostraciones alternativas a las originales y señalaba que algunos axiomas podían ser demostrados. Las ediciones de Clavius y de Barrow fueron las más importantes para Leibniz, porque las menciona con mucha frecuencia, aunque hemos verificado en las bibliotecas mencionadas y en sus manuscritos que también consultó otras muchas ediciones y comentarios de los Elementos, como las de J. Peletier (1557), Scheubelius (1558), Ramus (1559), Herlinus y Dasypodius (1571), Comandino (1572), Rhodius (1609), Grynaeus (1630), Hérigone (1639), Richard (1645), Tacquet (1654), Borelli (1658), Sturm (1661), Fabri (1669), Deschalles (1672), Prestet (1676), Arnauld (1678), Mercator (1678), Mourguez (1680), G.

\footnotetext{
16 Véase la correspondencia cruzada entre Huygens y Leibniz en M.S. de Mora (2015) 7B, pp.433-7. También véase la correspondencia completa Leibniz-Huygens de 167980 en la edición Gerhardt, y principalmente, el Apéndice a la carta de Leibniz del 9 septiembre 1679, en M.S. de Mora 2015, 7B, pp. 511-19. También, J. Echeverría (1979), H. Freudenthal (1972), A.E. Heath (1917).
}

17 Véase Echeverría (1988). 
Vitale (1680), etc. Entre esa diversidad de ediciones, que en ningún caso eran ediciones críticas en el sentido actual del término, sino más bien versiones, comentarios o glosas a los Elementos, nos encontramos con todo tipo de modificaciones de los textos originales de Euclides, así como con variaciones en las listas de definiciones, axiomas y postulados, y también en las proposiciones.

Por lo tanto, Leibniz conocía bien las críticas y los comentarios a los Elementos que se planteaban en su época. No obstante, su posición al respecto fue muy original: la tarea que emprendió consistió en revisar cada uno de los axiomas, postulados y demostraciones así como las definiciones, principalmente del Libro I. Para ilustrar el modo de trabajar Leibniz el libro de Euclides, mediante su nueva Geometría Situs, comentaremos a continuación algunos manuscritos suyos, fechados en $1676-77$, en 1679 , en 1680 y en $1683 " 18$.

En el manuscrito de 10 de agosto de $1679^{19}$, se ocupó en primer lugar de la definición de espacio y luego de la de punto, analizando y modificando la de Euclides ("punto es lo que no tiene partes"). En lugar de tomar las nociones de todo y partes como primitivas, Leibniz partió de la relación de congruencia y definió el punto, primero mediante nociones expresadas en palabras, luego mediante signos:

"Para tratar realmente todo esto por orden hemos de saber que la primera consideración es el espacio mismo, esto es, lo extenso puro absoluto: puro de toda materia y de movimiento, y también absoluto, esto es, ilimitado, puesto que contiene toda extensión. Por ello todos los puntos están en el mismo espacio y se pueden referir mutuamente los unos a los otros. Que este espacio sea alguna cosa distinta de la materia o sólo una aparición constante o fenómeno, no me referiré a ello en este lugar.

Lo siguiente a considerar es el punto, es decir aquello más simple entre todo lo que pertenece al espacio o la extensión; pues del mismo modo que el espacio contiene a la

18 Véase M.S. de Mora (2015), pp. 417-511.

19 Leibniz 1995, fragmento IX, pp. 142-223. 
extensión absoluta, así los puntos expresan lo que es máximamente limitado en la extensión, sin duda el simple situs De ahí se sigue que el punto es un mínimo y carece de partes, y todos los puntos son congruentes entre sí (es decir, pueden coincidir), por lo tanto son semejantes y, se podría decir, iguales) ${ }^{20}$.

Obsérvese que a partir de esa definición de punto Leibniz considera que se puede demostrar que todos los puntos son congruentes entre sí, lo cual significa a su vez que pueden coincidir: todos los puntos son iguales entre sí (Ibid.). La coincidencia o igualdad entre puntos es una propiedad geométrica que hay que demostrar a partir de la definición dada, en lugar de darla por supuesto de manera intuitiva, como hace Euclides. Este es la idea fuerza del proyecto leibniciano de revisión de los Elementos de Euclides: intentar demostrar muchas cosas que Euclides da por supuestas, y para ello recurrir a nuevas definiciones y axiomas geométricos, basados en la noción de situs.

Por supuesto, a lo largo de su vida Leibniz propuso otras definiciones de punto, algunas muy interesantes para su teoría final del espacio y el tiempo. Una de las más famosas está en su Carta a Bourget ${ }^{21}$, de mayo de 1715 , donde no sólo reflexionó sobre los puntos en el espacio, sino también en el tiempo:

"En cuanto a la sucesión, parecéis pensar, señor, que hay que concebir un primer instante fundamental, así como la unidad es el fundamento de los números, y como el punto es también el fundamento de la extensión: a eso podría responder que el instante es también el fundamento del tiempo, pero como no hay ningún punto en la naturaleza que sea fundamental con respecto a todos los demás puntos, y por así decir, la sede de Dios, igualmente no veo en absoluto que sea necesario concebir un instante principal. No obstante, reconozco que existe esa diferencia entre los in-

20 Leibniz, o.c., pp.150-152.

21 Véase de Mora (2015), pp. 535-538. 
stantes y los puntos, de modo que un punto del universo no tiene la ventaja de la prioridad de naturaleza con respecto a otro, y en cambio el instante precedente siempre tiene la ventaja de la prioridad, no sólo de tiempo, sino también de naturaleza sobre el instante siguiente. Pero no es necesario en absoluto que haya por ello un primer instante.

Punto es lo más simple entre todo lo que pertenece al espacio, lo máximamente limitado en la extensión. Pues si A., B., Y. son puntos y el signo $Y \gamma$ significa congruencia, y si fuera A.Y $Y$ Y B.Y $Y$ Y C.Y, entonces el lugar de todas la $Y$ sería un punto, es decir que no habría más que un $Y$ que lo satisfaría."

Como puede verse, en esta definición de 1715 Leibniz retoma algunas definiciones suyas que ya había usado en 1679.

En otro manuscrito inédito, éste de 1680, Leibniz contrapuso las nociones de espacio y punto:

"Espacio es lo que hay más simple en materia de extensión, el punto es lo que hay más simple en materia de situación"22.

Ahí tenemos otra de las ideas claves de las investigaciones leibnicianas en esa época. En lugar de basar la geometría en la noción de extensión, como Descartes, la geometría de Leibniz habría de basarse en la de situación: por eso sus indagaciones se orientaron hacia una topología de puntos, donde los lugares geométricos son definidos como conjuntos de puntos, no mediante ecuaciones.

Habría otras definiciones de punto a comentar, pero las dos mencionadas puede bastar para dar una cierta idea del proyecto leibniciano en 1679-1680. En general, Leibniz se dedicó en sus ensayos geométricos a probar qué se podría demostrar con unas y otras definiciones geométricas, y siempre en el contexto de su geometría de situación, es decir, intentando fundamentar la Geometría en el Analysis Situs.

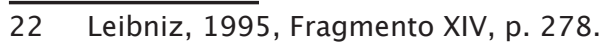


En cuanto a la definición euclidiana de línea, a Leibniz no le pareció suficientemente rigurosa. En un manuscrito inédito titulado Definitiones, que Bodemann ha fechado hacia 1685 , la consideró "más popular que geométrica" ${ }^{23} \mathrm{y}$, a continuación, propuso mejorarla mediante la siguiente definición:

"Es la vía de un punto. Es un trazo tal que dos partes arbitrarias no tienen más que un extremo común, que es un punto"24.

Este tipo de definiciones en las que se usa la noción de punto extremo, tras haber definido la de punto, son bastante frecuentes entre los escritos geométricos de Leibniz. También la definición euclidiana de recta le pareció oscura y propuso varias definiciones alternativas, por ejemplo las siguientes, todas ellas del manuscrito recién mencionado de 1685:

"Recta es aquella (línea) cuya magnitud es como la distancia entre sus extremos.

Es el extenso que se percibe por el hecho de que dos puntos son percibidos simultáneamente.

Es la trayectoria de un punto desplazado de forma que conserve una situación semejante en relación con los lugares que recorre.

Es aquella cuyas partes cualesquiera están en una situación semejante.

Es el trazo que no puede moverse si dos de sus puntos permanecen inmóviles"25.

Como puede verse, Leibniz solía ensayar con diferentes definiciones, conforme a su metodología de análisis de las definiciones, que puso en práctica una y otra vez a lo largo de su vida, tanto en lógica como en matemáticas. En un manuscrito de 1679, por ejemplo, abordó el problema de definir la noción de línea en base a la noción de congruencia, $(\gamma)$, que

23 Leibniz Handschriften XXXV, I, Nr. 5, BI. 19.

24 Ibid.

25 Ibid. 
durante ese año fue la relación geométrica principal en sus investigaciones, y lo hizo del modo siguiente:

"Sea la línea 1A 2A 3A etc. Sean congruentes B 1A $2 A$ etc. D. y $F 1 E 2 E$ etc. $G$, y coincidan $B$ y F. Coincidan igualmente $D$ y $G$, y coincidan del mismo modo los $A$ y sus correspondientes $E$, de suerte que BAD y FEG sean una y la misma línea. Decimos que esa línea es recta26",

y a continuación explicó que "hoc est quod voluit Euclides puncta rectae ex aequo jacere" 27 , lo cual deja claro su propósito: mejorar la definición de Euclides, aclarando la noción de "yacer por igual"28.

El proyecto leibniciano quedó expuesto con bastante amplitud en un largo manuscrito fechado en agosto de 1679, que a nuestro entender es el más importante de esta segunda época de investigaciones leibnicianas sobre la Geometria situs, en este caso con la denominación de Característica Geométrica ${ }^{29}$ :

“(...) Los caracteres algébricos no expresan todo lo que se debe considerar en el espacio (se supone que los Elementos ya han sido inventados y demostrados) y no significan el sitio propio de los puntos, pues se investigan las magnitudes dando muchos rodeos. Por lo que resulta muy difícil expresar mediante el cálculo lo que la figura muestra; y más difícil todavía producir en la figura lo que el cálculo obtiene: de ahí que las construcciones que el cálculo aporta son en su mayor parte extremadamente distorsionadas e incómodas.

26 L. Hd. XXXV, I, Nr. 2, 8 retro; fragmento 5 de la edición Echeverría 1984, p. 53.

27 Ibid., p. 54. La definición euclidiana de línea recta es la siguiente: "una línea recta es aquella que yace por igual respecto de los puntos que están en ella" (Euclides, 1991, p. 190).

28 Como comenta Luis Vega, el editor de la edición más fiable de los Elementos de Euclides en lengua española (Madrid, Gredos, 1991), esa definición de Euclides sólo fue mejorada tiempo después, cuando Arquímedes definió la recta como "la más corta de todas las líneas que tienen los mismos extremos" (Ibid., p. 191) en su libro Sobre la esfera $y$ el cilindro.

29 Leibniz, 1995, fragmento IX, ya mencionado anteriormente. 
Ciertamente observo que los geómetras suelen añadir a sus figuras algunas descripciones, con las que se explican las figuras, acerca de lo que con la figura misma no se puede conocer, como la igualdad de las líneas o la proporcionalidad, para que se puedan entender con el añadido de las palabras; la mayoría demasiado largas y utilizando muchas palabras para exponer lo que en la figura misma es manifiesto, bien para que el razonamiento sea más riguroso y no dependa de los sentidos ni de la imaginación, sino que todo sea traducido con el razonamiento; o bien para trazar las figuras a partir de su descripción de modo que si se perdieran, pudieran ser restituidas. Incluso aunque esto no se observe siempre exactamente, nos han provisto así de unos vestigios de Característica Geométrica" ${ }^{30}$.

Dicho fragmento del mes de agosto, escrito en latín y bastante largo, es uno de los ensayos escritos por Leibniz que acabaron generando el famoso anexo a la carta de Leibniz a Huygens de septiembre de 1679. Dicho documento fue escrito en francé $\mathrm{s}^{31}$ y representa la primera tentativa de comunicar su proyecto a otros matemáticos, en este caso por la vía de la correspondencia privada, como fue habitual en Leibniz. En dicho anexo presentó a Huygens un resumen de su Característica Geométrica de 1679, usando la relación de congruencia como noción geométrica básica. Partió únicamente de las nociones de punto y congruencia, definió primero el espacio infinito (todos los puntos congruentes con uno dado), luego la superficie de la esfera (añadiendo la noción de equidistancia, en términos de congruencias), luego el plano (conjunto de puntos que tienen la misma situación con dos puntos dados), luego la circunferencia y finalmente la noción de línea recta. Esta última definición es la siguiente:

"Es el lugar de todos los puntos $Y$ con respecto a los cuales tres puntos dados A. B. C. se comportan del mismo modo; todos esos puntos [se refiere a los que "se comportan del

30 Leibniz, o.c., p. 145.

31 Leibniz 1995, fragmento XII, pp. 257-265. 
mismo modo", es decir, a los que están en la mismarelación de congruencia con $\mathrm{A}, \mathrm{B}, \mathrm{y} \mathrm{C}$ ] caerán en la recta infinita" 32 .

Se trata de un planteamiento muy original para aquella época, porque definía los principales lugares geométricos de Euclides en base a una sola relación, la de congruencia, y no a intuiciones subyacentes a las figuras, lo cual mejora notablemente la estructura lógica de la Geometría Elemental. En el caso de la recta, la definió como el conjunto de puntos congruentes con tres puntos dados, en lugar de decir que es la línea que pasa por dos puntos, lo cual es muy intuitivo, pero poco riguroso. Esta es, en efecto, una de las críticas de Leibniz a Euclides: tenía claro que por dos puntos pasan muchas líneas, por ejemplo quebradas o curvas, no una sola, razón por la cual la definición de Euclides le parecía criticable, dada la oscuridad del concepto de "yacer igual". Por otra parte, una vez definida la recta como el lugar de los puntos congruentes con tres puntos dados, demostró fácilmente un primer teorema, la intersección de dos planos es una recta, únicamente en base a la relación de congruencia. Lamentablemente, Huygens no entendió bien el propósito de Leibniz, lo cual tampoco es de extrañar, puesto que el filósofo de Hannover estaba anticipando un par de siglos las investigaciones sobre los fundamentos de la Geometría. Al final, tras un par de cartas más con Huygens, pero sin llegar a explicar a fondo su proyecto, tuvo que desistir: Huygens no era el interlocutor ideal para ese tipo de planteamientos de fundamentación lógica de la Geometría. Ello no le impidió proseguir sus propias investigaciones. Así, en enero de 1680 redescubrió la definición arquimediana de recta, Recta es la vía mínima de un punto a otro punto, y para investigarla introdujo la relación de semejanza entre todo y partes, en lugar de la congruencia entre puntos (Recta es aquella cuya parte es semejante al todo). También enunció algún teorema (cualquier recta es semejante a cualquier otra), aunque sin llegar a una demostración rigurosa basada en caracteres, como la que le comunicó a Huygens ${ }^{33}$.

La mayoría de los escritos de Leibniz sobre la Geometría de Euclides y sus nociones básicas consisten en ensayos geométricos que indagan

\footnotetext{
$32 \quad$ Ibid., p. 264.

33 Véase Leibniz 1995, fragmento XV, pp. 288-289.
} 
nuevas fundamentaciones posibles de la geometría elemental. De hecho, no publicó ninguno de ellos, aunque sí dio alguna noticia de los mismos a ciertos corresponsales, por ejemplo a Bodenhausen. Lo interesante de esos ensayos es que el propósito y el método casi siempre es el mismo, al menos en la década 1675-1685, que es la que estamos analizando: recurrir a nociones geométricas básicas que fuesen distintas y más claras que las utilizadas por Euclides en los Elementos. El empeño de Leibniz durante estos años consistió en investigar las diversas definiciones de punto, línea, recta, etc., con el propósito de buscar el mejor sistema posible de definiciones geométricas, que mejorase al de Euclides, el cual le parecía defectuoso, por lo que a las definiciones respecta.

Volviendo al manuscrito de 1685, Definitiones, Leibniz afirmó que la definición euclidiana de ángulo ni es lo bastante clara ni suficientemente congruente en su formulación ("non satis clara est, imo nec congrua"), lo cual le llevó a sugerir una nueva definición en base a la medida del ángulo, que luego apenas desarrolló: Es la magnitud del arco de círculo ${ }^{34}$.

En cuanto a la Definición 15 de la edición de Clavius, la del círculo, le pareció admisible, pero también mejorable ("bona est, sed non satis perfecta") ${ }^{35}$. Otro tanto ocurrió con el resto de las definiciones de Euclides: también le parecieron aceptables, excepto la 16, la 17 y la 29 (numeradas siempre según la edición de Clavius de los Elementos, que era la que usaba para este tipo de investigaciones).

En ese mismo documento también se ocupó de los postulados de Euclides, los cuales le parecían en principio posibilidades aceptadas, o generadas: "nihil aliud sunt quam assumatae possibilitates, aut generationes". En el presente artículo no vamos a ocuparnos de los escritos de Leibniz sobre los postulados euclidianos, aunque los hay, como ya señalamos al principio.

Respecto a los axiomas hizo un comentario que es clave en su concepción de la geometría y en su crítica a Euclides, y que tiene un indudable interés filosófico e histórico: piensa que todos los axiomas (de Euclides) pueden ser demostrados, y que hacerlo sería una contribución a la cien-

34 Leibniz Handschriften XXXV, I, Nr. 5, BI. 19.

35 Ibid. 
cia mucho mayor de lo que se cree ("Puto omnia axiomata posse demonstrari, idque majoris esse ad perfectionem Scientiae, quam quis credat"). De hecho, continuó con esta tarea hasta sus últimos años, intentando demostrar la mayor parte de los axiomas. También se dedicó a corregir los defectos y las incorrecciones de Euclides en sus demostraciones y en los enunciados de algunos teoremas, y a veces también en las figuras.

Tras este somero examen de algunos de sus manuscritos criticando a Euclides, podemos concluir que Leibniz trató de realizar una revisión completa de los Elementos, y que ese fue un proyecto suyo coherente y constante, por más que aparezca diseminado entre sus manuscritos, borradores y artículos, la mayor parte inéditos. La edición monográfica y exhaustiva de este conjunto de fragmentos resultaría del máximo interés para conocer a Leibniz como geómetra y como filósofo de la Geometría, así como para conocer a fondo su concepción del Analysis Situs.

\section{La nueva Geometría de Leibniz}

Vale la pena hacer un extracto más detallado de las ideas de Leibniz acerca de su nueva Característica Geométrica, que dio origen al Analysis Situs. En el resumen adjunto a su carta a Huygens de septiembre de 1679, se mostró entusiasmado ante este nuevo descubrimiento:

"He encontrado algunos Elementos de Geometría totalmente nuevos y completamente diferentes del Algebra, pues ésta sería una característica de los números indeterminados o magnitudes, no expresa directamente la situación, los ángulos y el movimiento. El Álgebra está obligada a suponer los Elementos de Geometría, mientras que esta Característica "empuja el análisis hasta el final"36.

En su texto más amplio, de agosto del mismo año precisa:

"La utilidad de los caracteres consiste en que, mientras se opera con ellos, no es necesaria la cosa a la que representan hasta el final de la operación. Los caracteres utilizados

36 Leibniz 1995, fragmento 12, pp. 257-258. 
en el Álgebra y en la Geometría no expresan todo lo que se debe considerar en el espacio. Por lo que resulta muy difícil expresar mediante el cálculo lo que la figura muestra y más difícil todavía producir en la figura lo que el cálculo obtiene. ${ }^{37}$

Los caracteres o signos geométricos que introdujo Leibniz son letras para simbolizar los puntos de las figuras, expresando con su colocación o transposición diversas relaciones entre dichos puntos. Por otra parte, definió el movimiento, uno de sus conceptos más importantes, como la mutación continua del situs. Las relaciones entre los puntos son diversas, pero en esta época de la Geometría Situs primó la de congruencia: $A \gamma \gamma B$, aunque también tuvo en cuentas las relaciones de igualdad y de semejanza, las cuales, en el fondo aparecen como dos modalidades distintas de congruencia. En efecto, tanto lo semejante como lo igual es congruente.

En cuanto a la caracterización de la congruencia, la hizo en base a propiedades “lógicas", por así llamarlas. Por ejemplo, $A B$ Y $\curlyvee$ BA, porque dos puntos $A$ y $B$ pueden cambiar de lugar manteniendo el situs entre ellos. Dos cosas congruentes entre sí pueden aplicarse la una sobre la otra sin ninguna mutación en ellas, aunque no ocupen el mismo lugar. En cuanto a la igualdad, son iguales ( $\mathrm{a}$ b, escribe Leibniz), las cosas que tienen la misma magnitud, es decir, que se pueden considerar congruentes sin añadirles ni quitarles nada. Dos extensos, aunque no sean congruentes, pueden resultarlo sin modificación de su cantidad, si se realiza una transmutación de sus partes o puntos, y entonces se dice que son iguales. Si dos cosas a y b, son homogéneas no hay ninguna diferencia entre las mismas, las partes de la una son iguales a las partes de la otra. Ni a es mayor que b ni b es mayor que a, son iguales. Dos cosas son semejantes: $\mathrm{a} \sim \mathrm{b}$, si no pueden discernirse una de otra, tomándolas independientemente, solo se pueden discernir por co-percepción. Son semejantes las cosas que tienen proporcionalidad homóloga, o sea que conservan el mismo situs. En suma, al estudiar las propiedades y los teoremas que se derivan de estas relaciones geométricas básicas se

37 Ibid., fragmento 9, p. 145. 
puede establecer y demostrar la Característica Geométrica, y todo ello sin recurrir a figuras, sólo mediante signos, a diferencia de Euclides. Por tanto, al proyecto de Leibniz le subyace la idea de investigar la Geometría por una vía muy distinta a la de Viète y Descartes, a saber: como un análisis y un cálculo de la situación, que sea más general que la geometría de Euclides, y también que la de Descartes.

\section{Tres manuscritos de Leibniz por estudiar a fondo}

Para terminar, vamos a mencionar otros tres manuscritos inéditos sobre el mismo tema, también de la década en la que nos estamos centrando en este artículo: Demonstrationes Euclideas, ut a Clavio exhibentur, revocabimus... (hacia 1680), Prima Geometriae Principia (hacia 1683) y el Specimen Analyseos Figuratae in Elementis Geometriae (hacia 1683). En estos fragmentos se pueden encontrar nuevas tentativas de desarrollar el proyecto de Leibniz, así como algunos resultados que consiguió, pero también las dificultades que experimentó para llevar a cabo su objetivo. Los dos últimos manuscritos y el Circa Geometrica Generalia (ya editado en la revista Theoria $^{38}$ ) constituyen un conjunto realmente notable para comprender la complejidad del proyecto leibniziano de una nueva Geometría Situs, construida a partir del análisis previo de los Elementos de Euclides, fundamentalmente desde la óptica de las relaciones de congruencia entre puntos. El fragmento editado por Gerhardt, In Euclidis $\Pi \Omega$ OTA, es en nuestra opinión, menos preciso, a pesar de su redacción ulterior ${ }^{39}$.

En el fragmento Demonstrationes Euclideas analizó algunos axiomas ("Diameter circulum bifariam secat", "Duae rectae non habent segmentum communem", "Duae rectae in uno puncto concurrentes si producantur se in eo puncto intersecabunt", "Anguli recti sunt aequales", "Duae rectae spatium non comprehendunt") y también 25 proposiciones de la edición de Clavius del Libro I de los Elementos, siempre subrayando la existencia de presupuestos no explicitados por Euclides. Leibniz propuso en él algunas nociones geométricas nuevas, muy interesantes para

38 THEORIA 2, VI, Nr. 14-15 (oct. 1991), pp. 55-66.

39 Mathematische Schriften (ed. Gerhardt), V, pp. 183-211. 
demostrar los axiomas: por ejemplo, una notación para designar que un punto está situado entre otros dos. También puso de manifiesto que Euclides presupone implícitamente muchos otros axiomas, como el de la superposición.

En el fragmento Prima Geometriae Principia propuso una nueva fundamentación para la Geometría, al introducir la noción de determinación, así como el axioma: "si determinantia sint coincidentia, congrua, similia; talia erunt determinata" 40 . Este es un ensayo de su propia Geometría Situs, a la que siempre concibió como una ciencia mucho más general que la de Euclides, por estar basada en nuevos principios, concretamente en relaciones geométricas (congruencia, distancia, determinación, etc.), en lugar de partir de nociones geométricas aparentemente intuitivas (punto, recta, figura etc.), pero muy poco claras. La pretensión de Leibniz consiste siempre en proponer nuevas definiciones y demostraciones de las nociones geométricas, usando para ello nuevas notaciones "de palabras y caracteres".

El fragmento Specimen Analyseos Figuratae in Elementis Geometriae es sin duda el más interesante porque, al proponer sus propias definiciones, axiomas y postulados, abordó cuestiones a las que hay que calificar de topológicas, y no simplemente geométricas. Entre dichas nociones «primitivas», por ejemplo, introdujo las nociones de punto interior $y$ exterior a un círculo (definiciones 4 y 5), así como un nuevo axioma, el 2, realmente notable para la época: "Continuum quod intra et extra figuram est, eius circumferentiae occurrit" 41 . El axioma 1, en cambio, lo mantuvo en su formulación tradicional euclidiana, lo cual parece implicar que lo aceptó, al menos en este escrito: "Totum maius parte". Con su nuevo sistema de definiciones, postulados y axiomas, que difiere mucho del de Euclides, Leibniz consiguió construir demostraciones rigurosas de las 8 primeras proposiciones del Libro I de los Elementos. Todas ellas se orientan hacia una Geometría construida en parte en término conjuntistas, e incluso con algunos anticipos de lo que hoy en día se denomina Topología General, más que una Geometría métrica y algebrista como la de Descartes. A nuestro entender, la edición crítica de este último

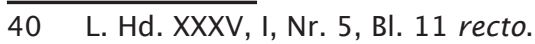

41 L. Hd. XXXV, I, Nr. 14, BI. 21 recto. 
manuscrito, así como las de los otros dos recién mencionados, resulta indispensable para tener una comprensión cabal del proyecto de Leibniz al criticar los Elementos de Euclides, y ello como vía para crear un Analysis Situs que vaya más allá de la geometría de las figuras geométricas y se convierta en un auténtico análisis de la situación.

Entre los manuscritos adicionales de Leibniz concernientes a los Elementos de Euclides, se encuentran asimismo algunas investigaciones sobre el postulado de las paralelas. Pero en nuestra opinión, la búsqueda de Leibniz va en otra dirección distinta a la de las geometrías no euclidianas. Su verdadera intención fue analizar y demostrar los axiomas y postulados geométricos de Euclides, en principio todos, no sólo el quinto postulado. Su reflexión nos parece mucho más actual, en este sentido, que la que se limita a estudiar el quinto postulado de Euclides, lo cual no es más que una parte de la inmensa tarea que propuso Leibniz: construir nuevas geometrías modificando las definiciones, los axiomas y los postulados.

Es necesario subrayar que los historiadores de las Matemáticas (prácticamente sin excepción), continúan ignorando esta revisión leibniziana de los Elementos de Euclides. Salvo las contribuciones de Knecht y de Münzenmayer y la tesis no publicada de Joseph Lauter, Leibniz continúa sin haber encontrado su lugar en la historiografía de los Elementos. En los últimos años, sin embargo, ha aparecido el excelente libro de Vincenzo De Risi, Geometry and Monadology. Leibniz's Analysis Situs and Philosophy of Space (2007). En ese trabajo, de Risi sostiene que el hilo conductor de estas investigaciones de Leibniz es el concepto de espacio, tanto en su ámbito matemático, el Analysis Situs, como en el metafísico, la Monadología. La argumentación de De Risi es convincente y complementa bien nuestra propia perspectiva. Leibniz prosiguió durante toda su vida con estas ideas y en los últimos años de su vida llegó a construir una auténtica teoría del espacio y el tiempo, como mostró claramente en su debate con Clarke. Por tanto, al final de su vida hay escritos de Leibniz sobre la Geometria Situs que expresan en profundidad sus concepciones filosóficas sobre el espacio y el tiempo. En los años de París y al comienzo de su época en Hannover, en cambio, sus investigaciones fueron ante todo geométricas y tomaron como punto de partida la lectura y el comentario crítico de los Elementos de Euclides. La aportación de De 
Risi es importante, porque analiza los últimos escritos de Leibniz sobre el situs, hasta ahora muchos de ellos ignorados.

En conclusión, diremos que la investigación de Leibniz hasta 1685 no tuvo como objetivo únicamente la reordenación de los Elementos, como era habitual en su época. Si se piensa en la clasificación propuesta por Luigi Maieru, en la que se consideran cuatro tipos de planteamientos respecto a los Elementos durante los siglos XVI y XVII, Leibniz no podría estar incluido en ninguno de ellos. El proyecto de Leibniz apunta a la construcción de una nueva Geometría, no sólo al perfeccionamiento de la estructura deductiva o racional de los Elementos. En suma: el proyecto leibniziano no admite ninguna comparación en su época, y probablemente habría que remontarse a los Grundlagen der Geometrie de Hilbert para encontrar algo semejante en la Historia de la Geometría.

\section{Bibliografía}

Alcantéra, J.P. (1993), "La caractéristique géométrique leibnizienne: travail du discernement et relations fondamentales", Revue d'Histoire des Sciences, XLVI, 4.

Bodemann, E. (1889), Der Briefwechseln des G.W. Leibniz, Hannover.

--- (1896), Die Leibniz-Handschriften, Hannover-Leipzig.

Borgato, M. T. (1988), "Unpublished manuscripts of Vitale Giordano, correspondant of Leibniz", V. Internationaler Leibniz-Kongress: Vorträge, Hannover, Leibniz-Gess, pp. 99-106.

Buseman, H. (1941), “On Leibniz's definition of planes", American Journal 63, pp. 101-111.

Couturat, L. (1901), La Logique de Leibniz, Paris: Alcan.

Duchesnau, F. (1993), Leibniz et la méthode de la science, Paris, PUF.

Echeverría, J. (1979), “L’Analyse Géométrique de Grassmann et ses rapports avec la Caractéristique Géométrique de Leibniz", Studia Leibnitiana XI/2, pp. 223-273.

--- (1984), Edition critique des manuscrits de Leibniz concernant la Caractéristique Géométrique en 1679, microfiche, Université de Lille III. 
--- (1982), "La Geometría leibniciana: de la Perspectiva al Analysis Situs", Actas II Congreso Sociedad Española de Historia de las Ciencias, Jaca, III, pp. 69-78.

--- (1988), "Géométrie et Topologie chez Leibniz", V. Internationaler Leibniz-Kongress: Vorträge, Hannover: Leibniz-Gesellschaft, pp. 213-220.

- (1991), "Cálculos geométricos de Leibniz", THEORIA 2,VI, 14-15, pp. 29-66.

- (1992), "La demostración de los axiomas de Euclides, según Leibniz", Revista Latinoamericana de Filosofía XVIII:1, pp. 33-41.

Euclides (1607), Euclidis Elementorum libri XV, accesit liber XVI, ed. Ch. Clavius, Coloniae 1591; $2^{\mathrm{a}}$ ed. Frankfurt, Hoffmann.

--- (1883-1888) Euclidis Elementa (ed. J. L. Heiberg), I-III, Leipzig.

--- (1991) Elementos (ed. Luis Vega), Madrid, Gredos.

Freudenthal, H. (1972), "Leibniz und die Analysis Situs", Studia Leibnitiana 4, pp. 61-69.

Giordano, V. (1680), Euclide Restituto, Roma.

Heath, A.E. (1917), "Hermann Grassmann. The neglect of his work. The geometric analysis and its connection with Leibniz's Characteristic", Monist 27, pp. 36-56.

Hecht, H. (1992, ed.), G.W. Leibniz im Philosophischen Diskurs über Geometrie und Erfahrung, Stuttgart, Teubner.

Hobbes, T. (1655), Elementorum Philosophiae sectio Prima De Corpore, Londini.

--- (1688) Opera Philosophica, 3 vols., Amsterdam.

Hofmann, J. Eh. (1949), Die Entwicklungsgeschichte der Leibnizschen Mathematik, Leibniz Verlag: München.

--- (1974), Leibniz à Paris 1672-76, London: Cambridge Univ. Press. Höppner, J. H. (1970), “Zur Datierung des Stückes 'De Calculus Situum', Studia Leibnitiana 2, pp. 233-235.

Knecht, H. H. (1974), "Leibniz et Euclide", Studia Leibnitiana 6, pp. 131-143. 
Lauter, J. (1953), Die Prinzipien und Methoden der Geometrie bei Leibniz, Diss. Naturwiss., Aachen.

Leibniz, G.W. (1923), Sämtliche Schriften und Briefe, Akademie-Ausgabe, Darmstadt-Berlin.

--- (1858-1863), Mathematische Schriften (ed. C.I. Gerhardt), Halle.

--- (1875-1890) Philosophische Schriften (ed. C.I. Gerhardt), Berlin.

--- (1899), Der Briefwechseln von G.W. Leibniz mit Mathematikern (ed. C.I. Gerhardt), Berlin.

--- (1903), Opuscules et fragments inédits (ed. L. Couturat), Paris.

--- (1995), La Caractéristique Géométrique (ed. J. Echeverría \& M. Parmentier), Paris, Vrin.

Maierù, L. (1982), "Il Quinto Postulato da C. Clavio a G. Saccheri”, Archive for the History of Exact Sciences 27, pp. 297-334.

Martin, D.J. (1983), Leibniz's Conception of Analysis Situs and its Relevance to the Problem of the Relationship between Mathematics and Philosophy, Ph.D., Emory University.

Mora, M.S. de (2014, ed.), Escritos Matemáticos de Leibniz, vol. 7A de la colección G. W. Leibniz. Obras Filosóficas y Científicas. Editorial Comares, Granada.

Mora, M.S. de (2105, ed.), Escritos Matemáticos de Leibniz, vol. 7B de la colección G. W. Leibniz. Obras Filosóficas y Científicas. Editorial Comares, Granada.

Müller, I. (1981), Philosophy of Mathematics and Deductive Structure in Euclid's Elements, Cambridge: MIT Press.

Münzenmayer, H. (1979), "Der Calculus Situs und die Grundlagen der Geometrie bei Leibniz", Studia Leibnitiana 11, pp. 274-300.

Pont, J.C. (1986), L'aventure des parallèles, Berne, P. Lang.

Ramée, P. de la (1659), Scholarum Mathematicarum libri XXXI, Basel.

Risi, V. de (2007), Geometry and Monadology. Leibniz's Analysis Situs and Philosophy of Space, Basel, Birkhäuser.

Solomon, G. (1989), Leibniz's Analysis Situs in Mathematical Context, Doctoral Diss., Univ. of Western Ontario, London. 
Taton, R. (1978), "L'initiation de Leibniz à la Géométrie", Studia Leibnitiana Supplementa XVII, pp. 103-129.

Wallwitz, G.G. (1991), "Strukturelle Probleme in Leibniz's Analysis Situs”, Studia Leibnitiana XXIII/1, pp. 111-118.

Weigel, E. (1662), Demostrationes Aristotelico-Euclideas, Lipsiae.

--- (1671), Idea Totius Encyclopediae Mathematico-Philosophici, Jenae. 